\title{
Hearing Characteristics and Doppler Shift Compensation in South Indian CF-FM Bats
}

\author{
Gerd Schuller* \\ Indo-German Project on Animal Behaviour, School of Biological Sciences, University of Madurai, Tamil Nadu, India, and \\ AK Neuro- und Rezeptorphysiologie, Fachbereich Biologie, Siesmayerstrasse 70, D-6000 Frankfurt/Main, \\ Federal Republic of Germany \\ Accepted August 6, 1980
}

Summary. 1. Echolocation pulses, Doppler shift compensation behaviour under laboratory conditions and frequency response characteristics of hearing were recorded in Rhinolophus rouxi, Hipposideros speoris and Hipposideros bicolor.

2. The frequencies of the constant frequency portions of the CF-FM pulses lie at about $82.8 \mathrm{kHz}$ for $R$. rouxi from Mahabaleshwar, at $85.2 \mathrm{kHz}$ for $R$, rouxi from Mysore. Hipposiderid bats have considerably higher frequencies at $135 \mathrm{kHz}$ in $H$. speoris and $154.5 \mathrm{kHz}$ in $H$, bicolor. The mean sound durations were $50 \mathrm{~ms}, 6.4 \mathrm{~ms}$ and $4.7 \mathrm{~ms}$, respectively.

3. $R$. rouxi compensates for Doppler shifts in a range up to typically $4 \mathrm{kHz}$ of positive Doppler shifts (Fig. 2). The Doppler shift compensation behaviour is almost identical to that of $R$. ferrumequinum.

4. H. speoris and $H$. bicolor do not compensate for Doppler shifts under laboratory conditions. Doppler shifts in the echoes induce emission frequency changes which are not correlated to the presented Doppler shifts (Fig. 3).

5 . The frequency response characteristics of hearing of $R$. rouxi show characteristic sensitivity changes near the bat's reference frequency as also found in $R$. ferrumequinum. The threshold differences between the low threshold at the reference frequency and a few hundred $\mathrm{Hz}$ below are 40 to $50 \mathrm{~dB}$ in awake bats (Fig. 5).

6. Frequency sensitivity changes near the emitted $\mathrm{CF}$-frequency of the bats are less pronounced in $H$. speoris or almost absent in H. bicolor.

* Supported by grants from Deutsche Forschungsgemeinschaft DFG Ne 146/9, Schu 390/1,2; the University Grants Commission of India, code No 430 and the Deutscher Akademischer Austauschdienst

Abbreviations: $C F$, constant frequency; $F M$, frequency modulated

\section{Introduction}

Several species of bats that do not belong to the same taxonomical group (rhinolophids, hipposiderids and the neotropical mormoopids) use echolocation signals composed of a relatively long-lasting constant frequency portion $(\mathrm{CF})$ terminated by a short final frequency modulated sweep (FM) to lower frequencies. Several special adaptations of the hearing system to the distinct pulse design, to the echolocation sound and to the behavoural strategies have been demonstrated in Rhinolophus ferrumequinum (Neuweiler, 1970; Schnitzler et al., 1976), Pteronotus p. parnellii (Suga, 1978) and hipposiderid bats (Grinnell and $\mathrm{Ha}$ giwara, 1972). One of the most striking adaptive features in Rhinolophus ferrumequinum and Pteronotus p. pamellit is the enhanced frequency sensitivity of hearing in the frequency range coinciding with the frequencies of the CF-portion of the emitted echolocation pulse and poor frequency sensitivity at adjacent lower frequencies (Neuweiler, 1970; Pollak et al., 1972). The other interesting behavioural adaptation in these bat species is the Doppler shift compensation leading to the decrease of the emitted frequency if a positive frequency shift occurs in the echo due to the relative movement between the bat and the reflector (Schnitzler, 1968; Schuller et al., 1974; Simmons, 1974).

Whereas the frequency pattern (CF-FM) of the echolocation signal is roughly the same in the three bat families, the pulses considerably differ in frequency and duration. The duration of the sound as well as the frequency may be important factors for the Doppler shift compensation behaviour, as sampling time and absolute frequency value affect the obtainable accuracy and extent of the compensation. A short stay in the laboratory of the Indo-German Project of Animal Behaviour at the University of Ma- 
durai, South India, offered the opportunity to investigate the echolocation signals, the Doppler shift compensation behaviour and the hearing capacities of the Indian Rhinolophus rouxi and two hipposiderid bat species, Hipposideros speoris and Hipposideros bicolor.

The results obtained on the sound structure, Doppler shift compensation and the frequency response characterisitics of hearing are presented in the following report.

\section{Methods}

The hipposiderid bats (H. speoris and $H$. bicolor) were caught in the Kila Kuyil Kudi rocks near Madurai University, the $R$. rouxi came from caves near Mahabaleshwar (about 200 miles south of Bombay) or roosting sites near Mysore.

The echolocation pulses were recorded from the hand-held bat about $30 \mathrm{~cm}$ in front of a quarter inch Bruel \& Kjær microphone (type 4135), amplified and stored on a Lennartz analog tape recorder. A double heterodyning technique was used to test the Doppler shift compensation behaviour. This technique has been extensively described in Schuller et al. (1974) and was modified only in selecting the mixing frequency and filter settings appropriate to the higher emitted CF-frequencies of the different bat species. Frequency distributions of the CF-frequencies in individual bats were obtained with a Nicolet 1070 laboratory computer (with plugin SH 71) used in its frequency distribution mode: the zero-crossings of the bat's echolocation signals were transformed in standard pulses that were counted during count gate times of $2 \mathrm{~ms}$ and $10 \mathrm{~ms}$ depending on the duration of the echolocation pulses and classified by frequencies. The count times mentioned above determine the frequency resolution to $500 \mathrm{~Hz}$ and $100 \mathrm{~Hz}$ respectively. As the count time intervals were not synchronized to the pulse emission, those at the beginning and the end of the pulses indicate lower frequencies than actually present in the echolocation pulse. Consequently the lower frequency part of the frequency distribution histograms will be slightly broadened which is insignificant for the evaluation of the Doppler shift compensation behaviour for which the maximum in the frequency distribution histogram is used.

Preparations of the animals for neurophysiological recordings were made with the conventional techniques as described earlier (Schuller, 1979). Gross silver or tungsten electrodes were used for recording the evoked potentials from surface and deeper layers of the inferior colliculus. The evoked potentials were averaged 32 times to determine the hearing thresholds with the Nicolet laboratory computer (Type 1070, plug-in SD-72/2A).

Anaesthetics (ether, Novocain) were only used during the surgical preparation of the animals but not during the recording sessions so that the recordings were made in the awake animal. During recording the wound margins were treated with local anaesthetics.

The acoustical stimuli consisted of tone bursts with a duration corresponding to the average duration of the echolocation pulses of the bats and had rise-decay times of $1 \mathrm{~ms}$. The tone bursts were delivered under free field conditions to the contralateral ear of the bat. The distance between bat and loudspeaker was $34 \mathrm{~cm}$. The frequency response of the loudspeaker varied by $\pm 5 \mathrm{~dB}$ in a frequency range from 30 to $150 \mathrm{kHz}$ and the thresholds were corrected accordingly.

The species were identified following the key given by Brosset in his work on bats of central and western India $(1962,1963)$.

\section{Results}

\section{A. Echolocation Pulse Characteristics}

The typical echolocation pulses of the three species $R$. rouxi, $H$. speoris and $H$. bicolor consist of a constant frequency portion (CF) with a short final frequency modulated sweep (FM). Frequencies of the $\mathrm{CF}$-portions and duration of the signal are different in the three species.

$R$. rouxi (specimens from Mahabaleshwar) emit a mean interindividual frequency of $82.8 \mathrm{kHz}$ (SD: $1.3 ; 6$ animals) in the $\mathrm{CF}$-portion and the echolocation pulses have a mean duration of $50 \mathrm{~ms}$ in resting animals. Bats from the same species, but from a site near Mysore (1,000 km away from Mahabaleshwar) had a higher interindividual mean CF-frequency at $85.2 \mathrm{kHz}$ (SD: $1.0 ; 6$ animals). The difference of the mean emitted CF-frequency in the two groups of bats

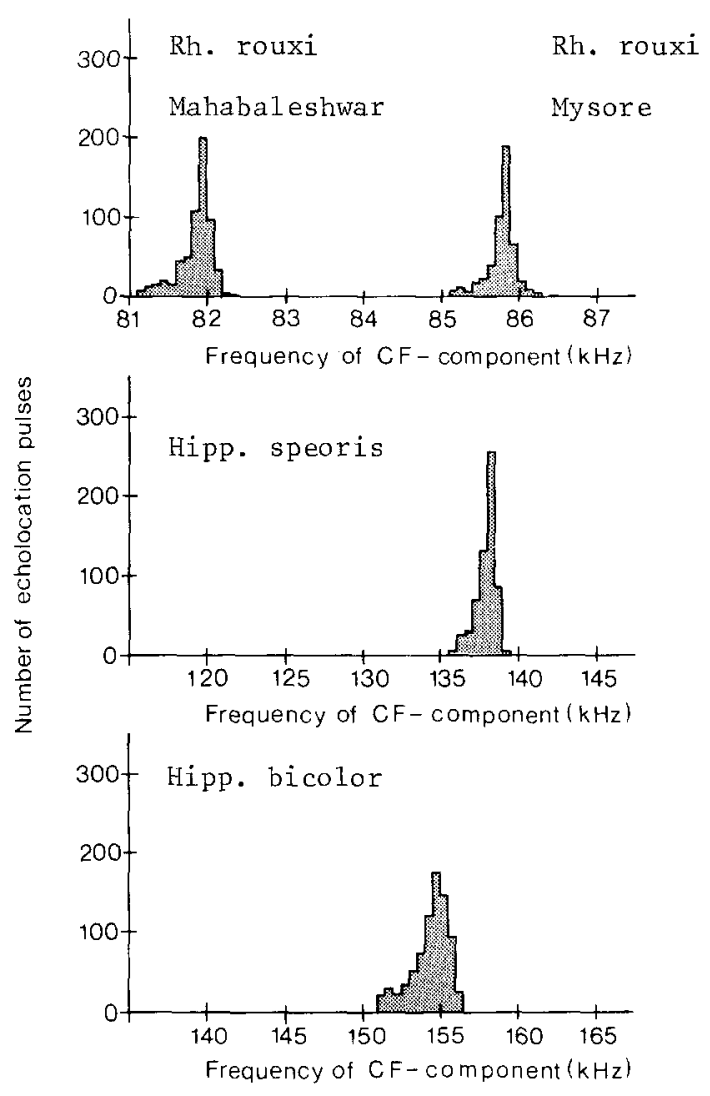

Fig. 1. Emission frequency in the constant frequency component (CF) of the bats Rhinolophus rouxi (upper), Hipposideros speoris (middle) and Hipposideros bicolor (lower). The histograms show the distribution of frequencies in the CF-portion for individual bats. Two different representative examples of CF-frequency distributions of $R h$. rouxi from Mahabaleshwar (left) and Mysore (right) are represented in the upper graph 
is $2.4 \mathrm{kHz}$. No difference in echolocation pulse duration could be detected in the two different groups of $R$. rouxi. The CF-frequency produced by the roosting bat is kept with high accuracy at the so called resting frequency. Examples of frequency distributions for two individual bats, one from Mysore and one from Mahabaleshwar, are shown in Fig. 1. The standard deviations in the individual bats ranged typically from $130 \mathrm{~Hz}$ to $230 \mathrm{~Hz}$, corresponding to an average frequency accuracy of $0.2 \%$.

The echolocation pulse of Hipposideros speoris and Hipposideros bicolor were shorter than those of $R$. rouxi and much higher in frequency. Most sound energy was emitted by $H$. speoris at a frequency of $135 \mathrm{kHz}$ (SD: $2.1 ; 10$ animals) and the CF-portion lasted between 6 and $7 \mathrm{~ms}$. H. bicolor maintained its echolocation frequency in the $\mathrm{CF}$-portion at $154.5 \mathrm{kHz}$ (SD: $2.3 ; 10$ animals) and the $\mathrm{CF}$-portion had durations between about 4 and $5 \mathrm{~ms}$. The frequency distributions in Fig. 1 show the accuracy with which the individual bats keep the emitted CF-frequency $(H$. speoris: $\mathrm{SD}: 660 \mathrm{~Hz}, H$. bicolor:

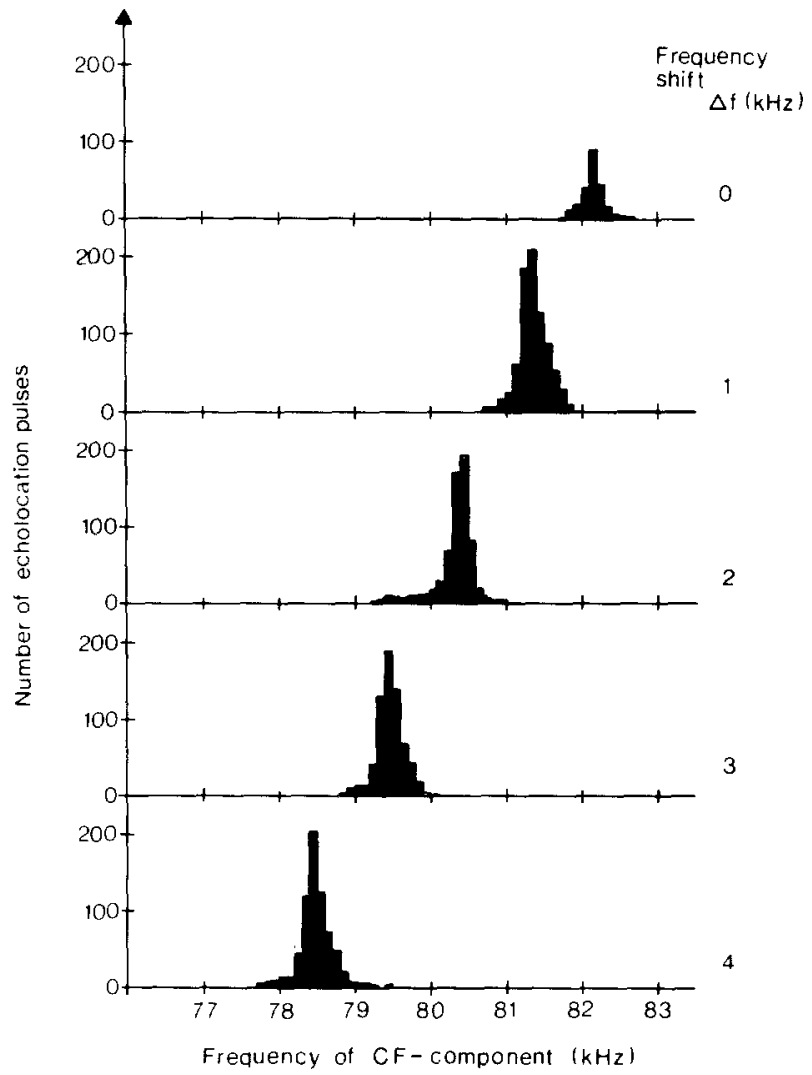

$\mathrm{SD}: 1.17 \mathrm{kHz}$ ). The variations of the $\mathrm{CF}$-frequencies are considerably higher for hipposiderid bats $(0.5 \%$ and $0.75 \%$ respectively) than for Rhinolophus $(0.2 \%)$.

\section{B. Doppler Shift Compensation Behaviour}

A bat flying at a certain speed towards the target receives echo frequencies that are shifted to higher frequencies due to the Doppler effect. Such echolocation situations can be electronically simulated by the playback of the emitted sounds that are electronically shifted to higher frequencies.

The Doppler shift simulation apparatus is the same as used for the investigations in $R$. ferrumequinum (Schuller et al., 1974). R. rouxi emitted its echolocation sounds spontaneously when sitting in front of the microphone-loudspeaker assembly. Its reaction to frequency shifted echoes is illustrated in Fig. 2, where the left graph shows the frequency distributions of the emitted CF-portions for different applied positive frequency shifts. The mean of each frequency distribution is depicted as a function of the Doppler
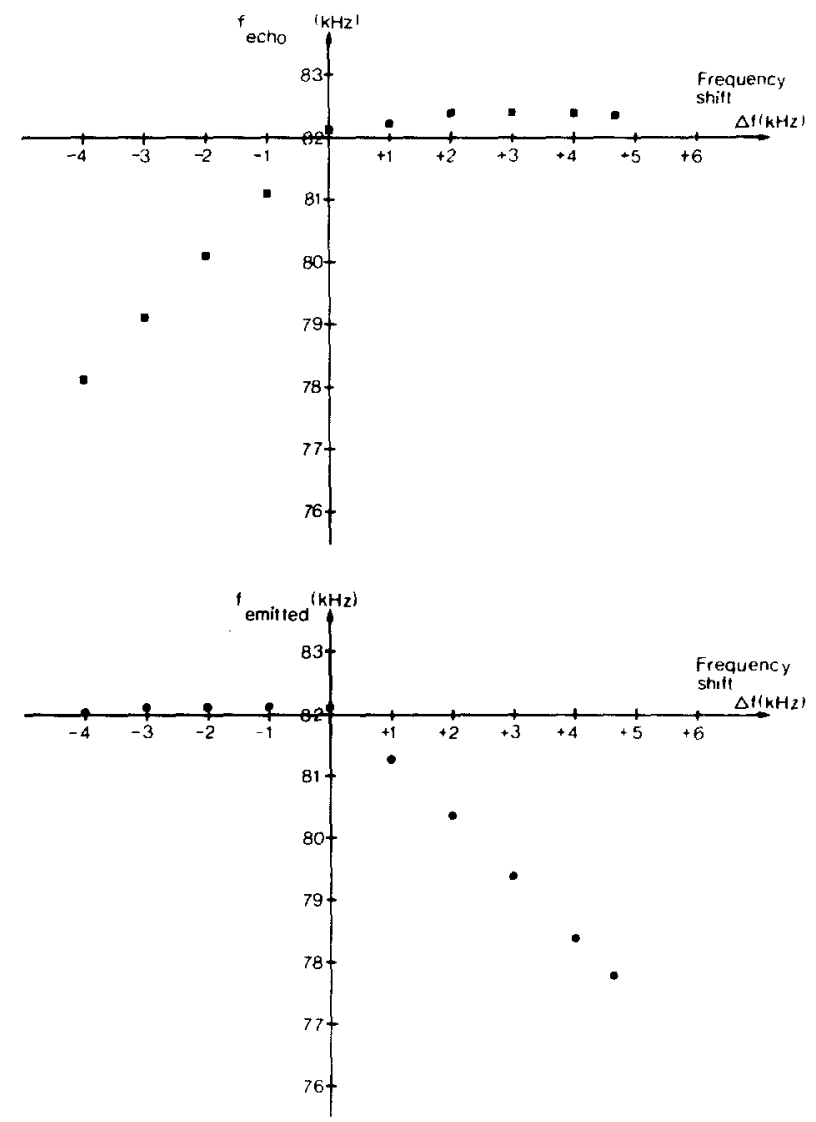

Fig. 2. Doppler shift compensation in Rhinolophus rouxi (Mahabaleshwar). Left: Distributions of emitted CF-frequencies for different frequency shifts from 0 to $4 \mathrm{kHz}$. Upper right: Echo frequencies heard by a bat exposed to Doppler shifts from -4 to $+4 \mathrm{kHz}$. Lower right: Emitted CF-frequencies of the bat when hearing Doppler shifts from -4 to $+4 \mathrm{kHz}$. The dots represent the mean value of the frequency distribution curves for the emitted CF-frequency of the echolocation sounds ( $\mathrm{N} \geqq 200$ sounds) 
shifts played back to the bat in the lower right graph. It can be seen that the bat compensates for the Doppler shifts heard in the echoes by lowering the emitted frequency. The compensation does not reach complete cancellation of the frequency shifts, but is incomplete by a compensation shift offset of about $150-200 \mathrm{~Hz}$. Consequently the echo frequency is slightly higher by this offset than the resting frequency. The frequencies actually heard by the bat after compensation are shown in the upper right graph of the figure. The average echo frequency after compensation has been called reference frequency (Schuller et al., 1974). Negative frequency shifts corresponding to increasing distance between bat and target are not responded to by any change in the emitted frequency of the CF-portion. The general characteristics of compensation for frequency shifts in $R$. rouxi are well described by the example in Fig. 2.

The frequency shift range within which proper compensation occurred varied between 3 and $4.5 \mathrm{kHz}$ and compensation offset ranged from 100 to $300 \mathrm{~Hz}$ in the 9 animals tested.

The Doppler shift compensation behaviour in $R$. rouxi can be considered as almost identical to that of $R$. ferrumequinum in all aspects so far investigated.

Six $H$. speoris and seven $H$. bicolor have been exposed to frequency shifted echoes. Most of the bats spontaneously emitted orientation pulses when sitting in the animal holder, others had to be stimulated to emit echolocation pulses by slightly touching their fur. None of the $H$. speoris showed clear compensation of the introduced Doppler shifts. They either did not react at all or emitted the orientation pulses with CF-frequencies different from the resting frequency but not tightly correlated to the Doppler shifts. In general the distributions of emitted frequencies broadened when the Doppler shifts were introduced in the echoes. Figure 3 shows the reaction of one $H$. speoris to Doppler shifts (upper graph).

The same was found in $H$. bicolor, when hearing Doppler shifted echoes. The bat again did not respond in a correlated way to the frequency shifts introduced in the echoes (lower graph in Fig. 3). However, both species showed larger variations of the emitted frequencies when exposed to Doppler shifts in the echoes compared to the variations of the resting frequency.

One bat was caught that corresponded in its taxonomical characteristics to the $H$. bicolor but differed in the colour of its fur, which was yellowish-orange in contrast to the grey fur of the other bats from this species. Brosset in his work on Indian bats (1962) describes different subspecies of $H$. bicolor, but it could not be determined whether the bat with the different fur colour belonged to another subspecies.

From observations during the captivity of the bat
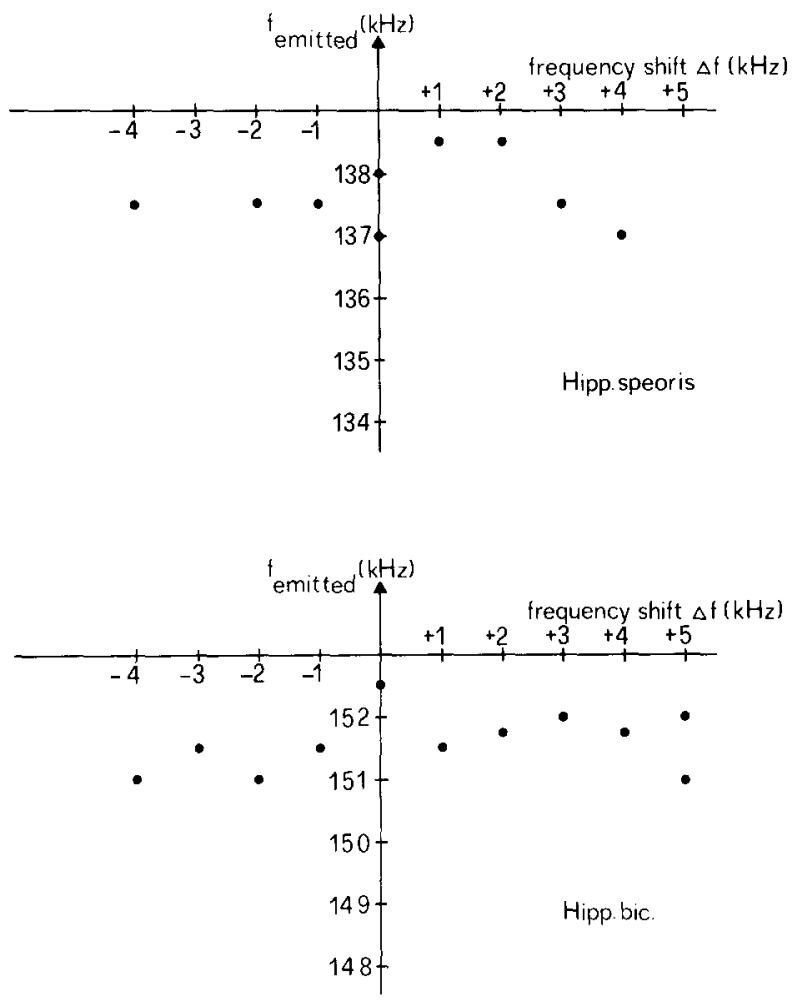

Fig. 3. Doppler shift compensation in Hipposideros speoris and Hipposideros bicolor. Emitted CF-frequencies of $H$. speoris (upper) and $H$. bicolor (lower) in response to frequency shifts between $-4 \mathrm{kHz}$ and $+5 \mathrm{kHz}$. The dots represent the mean value of the frequency distribution curves for the emitted CF-frequency of the echolocation sounds ( $\mathrm{N} \geqq 200$ sounds)

it was found to be in general more active and more skilled in catching wing beating moths than the grey variety of bats. The frequency of the CF-portion of this bat was $158 \mathrm{kHz}$, i.e., slightly higher than the means frequency of the $H$. bicolor $(154.5 \mathrm{kHz})$.

When the bat was tested in the Doppler shift playback apparatus it spontaneously emitted echolocation pulses and compensated frequency shifts up to $8 \mathrm{kHz}$. In Fig. 4 the Doppler shift compensation response of this bat is shown. The response is not as consistent as in $R$. rouxi and the accuracy of maintaining the lowered frequency at the compensated level is much reduced and consequently the frequency distribution broader. As the bat emits about double the frequency of that of $R$. rouxi the frequency shifts that this hipposiderid bat receives at a given flight speed are also twice as high as that in rhinolophid bats. Assuming that both species cover the same flight speed range, the range for Doppler shift compensation should be two times larger in the hipposiderid as compared to that of the rhinolophid bat. The result strikingly confirms this prediction: the Doppler shift compensation system of the hipposiderid bat covered frequency shifts up to $8-9 \mathrm{kHz}$. Unfortunately no further speci- 

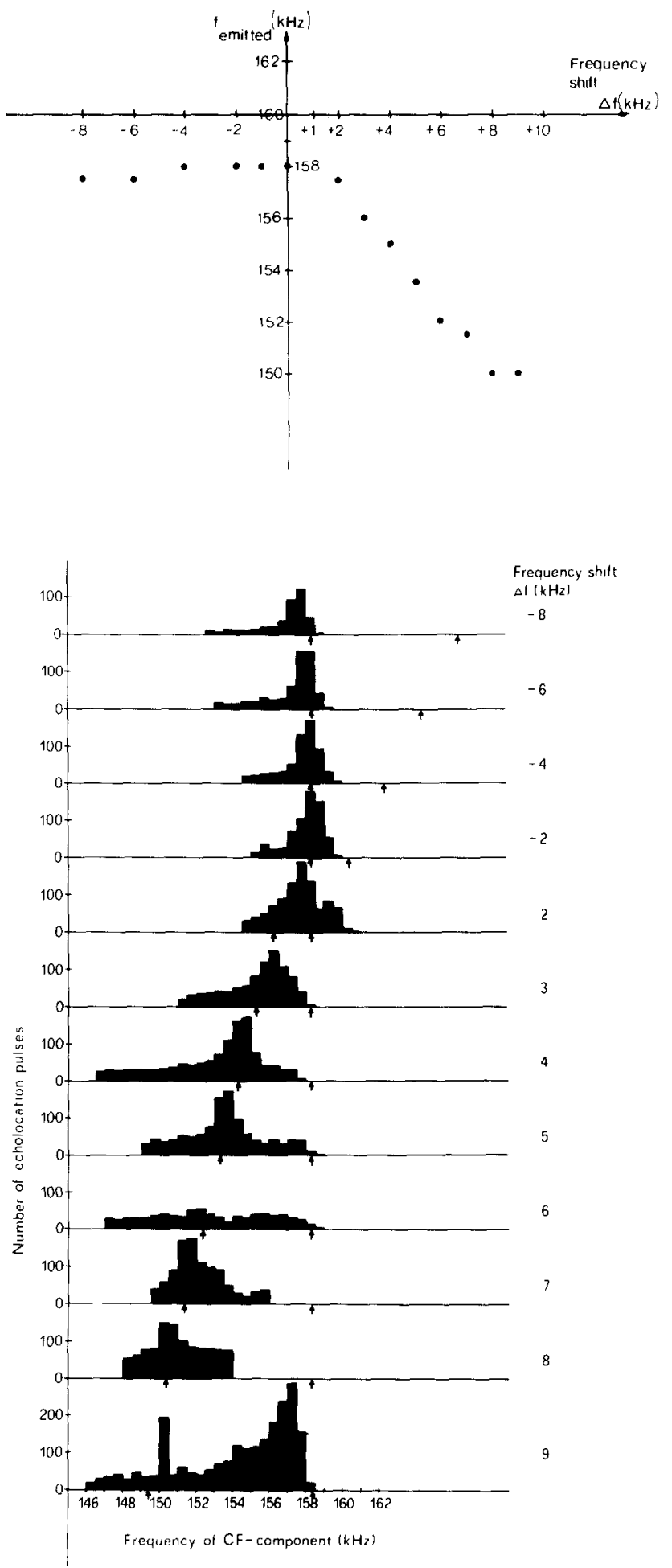

Fig. 4. Doppler Shift compensation of one Hipposideros bicolor (yellowish-orange fur). Left: Distribution of emitted CF-frequencies in response to Doppler shifts from -8 to $+9 \mathrm{kHz}$. Right: Emitted CF-frequencies of the bat when compensating for Doppler shifts between -8 and $+9 \mathrm{kHz}$ mens of the orange fur type were available, so that these results have to be considered as preliminary.

\section{Frequency Response Characteristics of Hearing as Determined with Evoked Potentials in the Inferior Colliculus}

The frequency response characteristics of hearing of $R$. rouxi, $H$. speoris and $H$. bicolor were determined using evoked potential recordings from the surface or from the center of the inferior colliculus. Threshold curves for evoked potential on-responses and off-responses from individual bats are represented in Fig. 5. The on-response threshold curves for $R$. rouxi (Fig. 5, left vertical row) had the characteristic low threshold range at the reference frequency with a steep increase of thresholds to lower frequencies (typically $50 \mathrm{~dB} /$ $100 \mathrm{~Hz}$ ) and a less abrupt increase in threshold on the high frequency side of the reference frequency (typically $5 \mathrm{~dB} / 100 \mathrm{~Hz}$ ). The threshold curve reaches a maximum at a frequency that is between 0.5 to $1.5 \mathrm{kHz}$ lower than the reference frequency and drops back to a threshold level comparable to that at the reference frequency at about $5 \mathrm{kHz}$ below the reference frequency. The difference between the maximum threshold and the threshold at the reference frequency ranges between 40 and $50 \mathrm{~dB}$ in awake bats. In $R$. rouxi off-responses were also obtained with stimuli having rise-decay times of $1 \mathrm{~ms}$. The off-response threshold curves had their minimum some hundred Hertz below the reference frequency and coincided with the resting frequency of the bat. The off-response threshold minimum is extremely narrow as can be seen from the graph (Fig. 5, left vertical row, triangles).

Near $42-43 \mathrm{kHz}$, i.e. half of the reference frequency of the bat, is another maximum of the hearing threshold which is much broader in frequency and less pronounced compared to the neighboring thresholds (threshold differences about $20 \mathrm{~dB}$ ).

The frequency response characteristics of hearing of $R$. rouxi correspond in all details and fine structural elements to that of the European $R$. ferrumequinum.

H. speoris (Fig. 5, middle row) showed much less characteristic threshold differences near its resting frequency. The largest threshold difference found between the minimal and maximal threshold over a narrow frequency band around the resting frequency was at the most $20 \mathrm{~dB}$ compared to $50 \mathrm{~dB}$ in $R$. rouxi. The narrow range of low threshold separating the $\mathrm{CF}$-hearing area from the rest of the audiogram is far less pronounced in hipposiderids.

Furthermore the minimum threshold in the audiogram did not coincide with the resting frequency but 

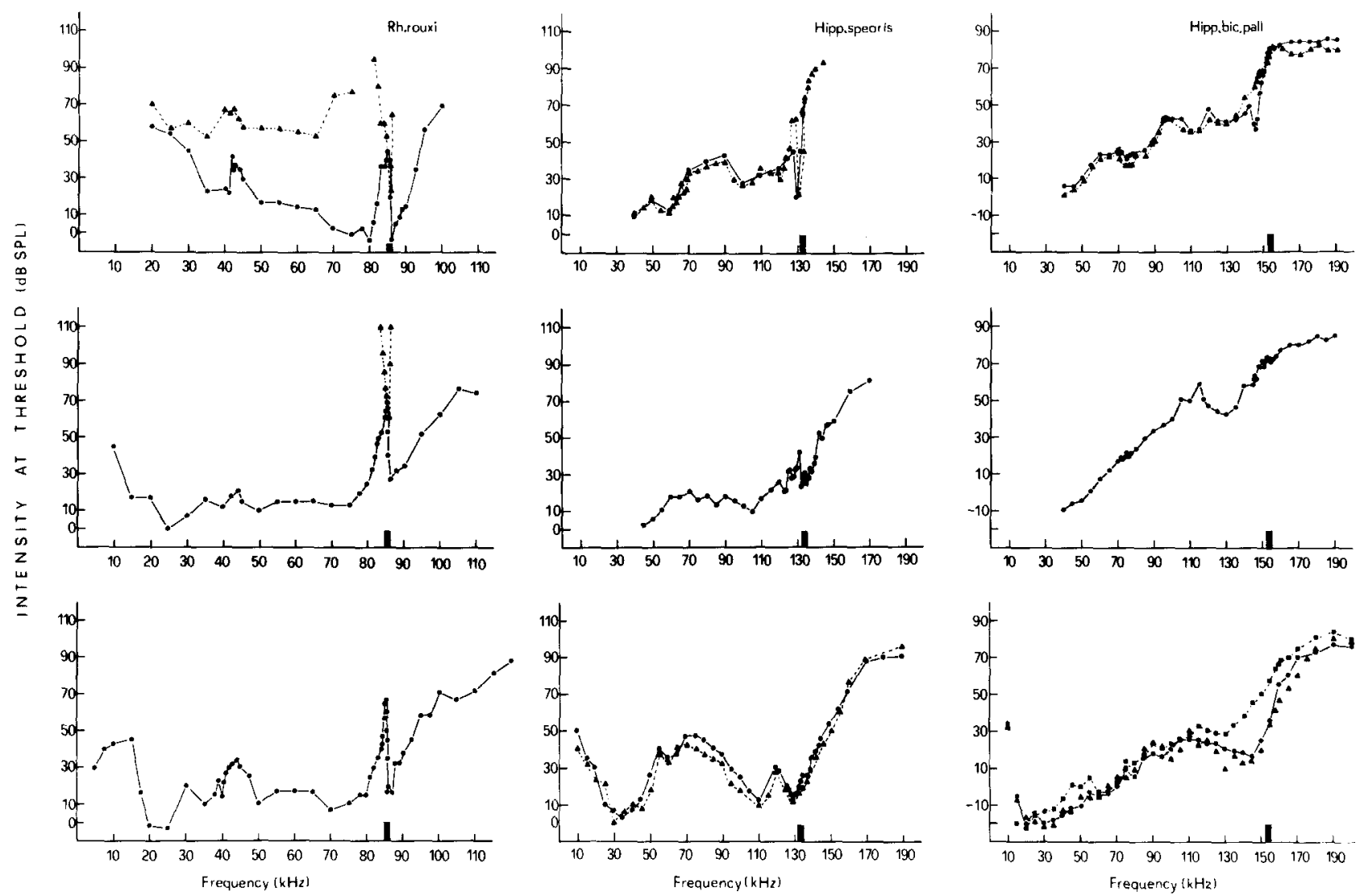

Fig. 5. Frequency response characteristics of hearing of three CF-FM bat species. On-response threshold curves for Rhinolophus rouxi (left row), Hipposideros speoris (middle row) and Hipposideros bicolor (right row). Bars on the abscissa: resting frequencies of the bats. Triangles in the first row: off-response threshold curves. Different symbols in the second and third row: threshold curves from different depths in the inferior colliculus

was always about $5 \mathrm{kHz}$ below (Fig. 5). The threshold curves of $H$. bicolor (Fig. 5, right vertical row) are even less structured and show a shallow low of thresholds for frequencies 20 to $30 \mathrm{kHz}$ below the resting frequency. In our sample of $H$. bicolor no threshold curve exhibited a narrowly tuned area around the resting frequency of the bat.

In $H$. speoris and $H$. bicolor no off-responses were obtained with stimulus rise-decay times of $1 \mathrm{~ms}$ and intensity levels of up to $100 \mathrm{~dB}$ SPL.

Unfortunately we could not obtain any threshold curves from the $H$. bicolor with the orange fur that compensated for Doppler shifts as the animal died and no further specimen were available.

\section{Discussion}

Rhinolophus rouxi, a species found in South India but having its distribution also in West and North India (Brosset, 1962, 1963), was found to possess an almost identical echolocation system to that of the European
Rhinolophus ferrumequinum. The structure of the echolocation signal, consisting of a long CF-portion with a final frequency modulated component, corresponds in duration and frequency to that of Rhinolophus ferrumequinum. There may be slight differences in the frequency of the CF-portion within a range of a few $\mathrm{kHz}$, which is even found among individuals of each species.

Both species are very vocal and spontaneously emit echolocation pulses when sitting in small but comfortable animal holders. In $R$. rouxi playback of Doppler shifted echoes to the bat induces Doppler shift compensation with the same characteristic range of about $4 \mathrm{kHz}$, the same accuracy in the frequency distributions and the same compensation offsets as found for $R$. ferrumequinum (Schuller et al., 1974). In this respect no differences in Doppler shift compensation behaviour as tested in the laboratory could be found in the two different species.

Determining the hearing characteristics of $R$. rouxi yielded curves that strikingly resemble those of $R$. ferrumequinum (Neuweiler, 1970). The threshold 
curves exhibit the characteristic low thresholds at the reference frequency with the extremely sharp slope of threshold increase within a few hundred $\mathrm{Hz}$ below and above the reference frequency $(50 \mathrm{~dB} / 100 \mathrm{~Hz}$ and $5 \mathrm{~dB} / 100 \mathrm{~Hz}$ respectively). The acoustical filter effect near the reference frequency is therefore a consequence of the steep increase of thresholds at both sides of the threshold minimum near $83 \mathrm{kHz}$.

The threshold curves correspond in all details to those of $R$. ferrumequinum if the position of resting and reference frequency in relation to the hearing threshold curve is considered. The reference frequency always coincides with the minimum of the threshold curve, whereas the resting frequency $(100$ to $300 \mathrm{~Hz}$ lower than the reference frequency) falls into the steep slope of rising threshold curve and in the minimum of the threshold curve for the off-responses. The species $R$. rouxi can be considered as having an echolocation system that is identical in its properties of echolocation signal, Doppler shift compensation and frequency response characteristics of hearing to that of $R$. ferrumequinum.

One interesting difference between the two groups of $R$. rouxi caught at different locations (Mahabaleshwar and Mysore) seems to be noteworthy. The resting frequencies of the two bat groups were significantly different by $2.4 \mathrm{kHz}$ which is quite large compared with the individual variation of resting frequency. All audiograms in this paper were measured in bats from Mysore and had their threshold minimum around $85.2 \mathrm{kHz}$. It can be expected that bats from Mahabaleshwar would have the threshold minimum at about $82.8 \mathrm{kHz}$. The second group of species of CF-FMbats investigated in this study, $H$, speoris and $H$. bicolor use a much shorter echolocation pulse at considerably higher frequencies. With the exception of one bat, no hipposiderid bat could be induced to Doppler compensate for frequency shifts presented with the Doppler shift simulation device. The North African hipposiderid bat, Asellia tridens (CF: $104 \mathrm{kHz}$ ) has been shown to compensate for flight induced Doppler shifts (Gustafson and Schnitzler, 1979). No other tests of Doppler shift compensation in hipposiderid bats are known. On the basis of the present laboratory experiments it cannot be completely excluded that hipposiderid bats do compensate for Doppler shifts when they are flying. Although the bats in our experiments emitted orientation sounds and received shifted echoes, some other factors such as the lack of flight movement or the lack of any change in delay time between the shifted echo and the outgoing pulse may prevent if from compensating properly in the Doppler shift simulation device. Further tests on flying bats must be performed to clarify this question.

One theoretical consideration may be given here: in $R$. ferrumequinum it was found that Doppler shift compensation only functions properly if the echo had a minimum duration of about $10 \mathrm{~ms}$ (Schuller, 1977). During the $10 \mathrm{~ms}$ the bat hears about 800 cycles of the echo signal. Assuming that for the fine frequency analysis for Doppler shift compensation purposes the bat would need about 800 cycles in the returning echo, the minimum pulse length should be $5 \mathrm{~ms}$ in $H$. bicolor and $5.9 \mathrm{~ms}$ in $H$.speoris. The respective mean pulse durations are $4.7 \mathrm{~ms}$ and $6.4 \mathrm{~ms}$, so that the bats would operate at the lower limit of Doppler shift compensation performance. In Asellia tridens the minimum necessary pulse length would be $6.6 \mathrm{~ms}$. This bat emits echolocation pulses during search flight of $8-10 \mathrm{~ms}$ (Gustafson and Schnitzler, 1979) and lie therefore well above the postulated pulse duration minimum for Doppler shift compensation.

In $H$. speoris an increase in frequency sensitivity and corresponding low in the audiogram near the emitted CF-frequency of the bats occurred at frequencies below the resting frequency. This is in contrast to the location of the threshold low in $R$. rouxi, where the frequency of the minimum is always a few hundred $\mathrm{Hz}$ higher than the resting frequency.

$H$. bicolor showed no pronounced frequency sensitivity changes which were tightly correlated to the $\mathrm{CF}$-frequency of the bats. The frequency characteristics of hearing of $H$. speoris and $H$. bicolor correspond to those found in other hipposiderid bats (Grinnell and Hagiwara, 1972). In contrast to the findings of Grinnell and Hagiwara we did not commonly find off-responses in hipposiderid bats which is probably due to rise-decay times of at least $1 \mathrm{~ms}$ of the tone bursts used in this study. Grinnell and Hagiwara indicate that off-responses are not detectable if the risedecay time is $1.5-2 \mathrm{~ms}$.

One exception to the lack of Doppler shift compensation in hipposiderid bats under laboratory conditions was found, i.e., a $H$. bicolor that had a yellowish-orange fur instead of a grey fur and a CF-frequency of $158 \mathrm{kHz}$. At this frequency and a given speed to the target this bat receives about double the shifts in the echo frequency as Rhinolophus does under the same conditions ( $\mathrm{CF}: 83 \mathrm{kHz}$ ). Assuming that both species fly and hunt at about the same flight speeds, the hipposiderid bats should have a range of Doppler shift compensation about double that of a rhinolophid bat. The striking result was that in this bat (yellowish-orange hipposiderid) the Doppler shift compensation range was $8-9 \mathrm{kHz}$, indeed, double that of Rhinolophus $(4-5 \mathrm{kHz})$.

The results on Doppler shift compensation behaviour in $H$. speoris and $H$. bicolor are not yet conclusive and need further elucidation by behavioural experiments. The echolocation system of the hipposiderid 
bats seems to be less adapted to Doppler shift compensation because of the short duration of the echolocation pulses and the little or absent specialization in the frequency characteristics of hearing around the resting frequency.

I wish to thank Prof. Krishnaswami and Dr. Chandrashekaran for their kind support of this work during my stay at the School of Biological Sciences, University of Madurai (India). The support of the University Grants Commission of the Government of India is gratefully acknowledged. The technical assistance of Ms. H. Hahn and Ms. A. Polotzek was most helpful.

\section{References}

Brosset, A.: The bats of central and western India (Part II). J. Bombay Nat. Hist. Soc. 59(2), 583-624 (1962)

Brosset, A.: The bats of central and western India (Part IV). J. Bombay Nat. Hist. Soc. 60(2), 337-355 (1963)

Grinnell, A.D., Hagiwara, S.: Adaptations of the auditory nervous system for echolocation. Studies of New Guinea bats. Z. Vergl. Physiol. 76, 41-81 (1972)

Gustafson, Y., Schnitzler, H.-U.: Echolocation and obstacle avoidance in the hipposiderid bat Asellia tridens. J. Comp. Physiol. 131, 161-167 (1979)
Neuweiler, G. : Neurophysiologische Untersuchungen zum Echoortungssystem der Großen Hufeisennase Rhinolophus ferrumequinum. Z. Vergl. Physiol. 67, 273-306 (1970)

Pollak, G., Henson, O.W., Novick, A. : Cochlear microphonic audiograms in the pure tone bat Chilonycteris parnellii. Science 176, 66-68 (1972)

Schnitzler, H.-U.: Die Ultraschallortungslaute der Hufeisen-Fledermäuse (Chiroptera-Rhinolophidae) in verschiedenen Orientierungssituationen. Z. Vergl. Physiol. 57, 376-408 (1968)

Schnitzler, H.-U., Suga, N., Simmons, J.A.: Peripheral auditory tuning for fine frequency analysis by the CF-FM bat, Rhinolophus ferrumequinum. III. Cochlear microphonics and N1-responses. J. Comp. Physiol. 82, 93-102 (1976)

Schuller, G.: Echo delay and overlap with emitted orientation sounds and Doppler shift compensation in the bat, Rhinolophus ferrumequinum. J. Comp. Physiol. 114, 103-114 (1977)

Schuller, G.: Coding of small sinusoidal frequency and amplitude modulations in the inferior colliculus of CF-FM bat, Rhinolophus ferrumequinum. Exp. Brain Res. 34, 117-132 (1979)

Schuller, G., Beuter, K., Schnitzler, H.-U.: Response to frequencies shifted artificial echoes in the bat, Rhinolophus ferrumequinum. J. Comp. Physiol. 89, 275-286 (1974)

Simmons, J.A.: Response of Doppler echolocation system in the bat, Rhinolophus ferrumequinum. J. Acoust. Soc. Am. 56, 672682 (1974)

Suga, N.: Specialization of the auditory system for reception and processing of species-specific sounds. Fed. Proc. 37, 2342-2354 (1978) 